Z. klin. Chem. u. klin. Biochem.

8. Jg., S. 231-234, Mai 1970

\title{
Einfacher in-vivo Nachweis Barbiturat-induzierter Hydroxylasen mit Hilfe von Tritium-markierten Steroiden als Indikatoren')
}

\author{
Von M. Wenzel, G. Köster und Jutta Hönicke \\ Biochemische und Isotopen-Abteilung, Pharmazeutisches Institut der Freien Universität Berlin
}

(Eingegangen am 23. Dezember 1969)

In dieser Arbeit wird ein allgemein anwendbarer in-vivo Nachweis für Hydroxylierungen beschrieben, der auf einer Analyse des TritiumAnteils im Körperwasser nach Injektion geeigneter Tritium-markierter Substrate beruht. Damit wird das Prinzip der Stoffwechsellabilen Tritium-Markierung auf eine neue Reaktion angewendet. Als Versuchstiere dienten Ratten, bei denen durch Gabe von Phenobarbital die Bildung von Hydroxylasen induziert werden kann. Nach Injektion von Ostradiol-[6,7- $\left.{ }^{3} \mathrm{H}\right]$ oder Testosteron-[7- $\left.{ }^{3} \mathrm{H}\right]$ findet man bei männlichen und weiblichen Ratten nur einen sehr geringen Anteil der injizierten Aktivität (2-3\%) im Körperwasser der Tiere als Tritiumwasser.

Nach Vorbehandlung der Tiere mit Barbiturat steigt dieser Anteil bei Weibchen auf das 5 fache und bei Männchen auf das 4 fache der Kontrollwerte. Unter gleichen Versuchsbedingungen steigt die HTO-Bildung bei Äthinylöstradiol- $\left[6,7-{ }^{3} \mathrm{H}\right]$ von 0,8 auf $3 \%$.

Dieser Effekt wird auf die Induktion von Hydroxylasen nach Phenobarbital-Vorbehandlung zurückgeführt. Anwendungsmöglichkeiten dieser in-vivo Methode werden diskutiert.

\section{The simple in vivo determination of barbiturate-induced bydroxylases with the aid of tritium-labelled steroids}

A generally applicable in vivo method is described for the determination of hydroxylation. It is based on an analysis of tritium in the body water, following the injection of suitable tritium-labelled substrates; thus the principle of metabolically labile tritium is applied to a new reaction. The experimental animals were tats, in which the administration of phenobarbital can be used to induce the formation of hydroxylases. After the injection of oestradiol- $\left[6,7-{ }^{3} \mathrm{H}\right]$ or testosterone- $\left[7-{ }^{3} \mathrm{H}\right]$ into male of female rats only a very small proportion of the injected activity $(2-3 \%)$ is found as tritium water in the body water of the animals.

After pretreatment of the animals with barbiturate, this proportion increases 5 fold in females and 4 fold in males compared with the control values. Under the same experimental conditions, the formation of HTO from ethinyloestradiol- $\left[6,7-{ }^{3} \mathrm{H}\right]$ increases from 0.8 to $3 \%$.

This effect is attributed to the induction of hydroxylases by the treatment with phenobarbital. Possible uses for this in vivo method are discussed.

Die Wirkung vieler Arzneimittel wird durch deren Abbaugeschwindigkeit im Organismus bestimmt. Von einer Anzahl von Pharmaka ist bekannt, daß sie - bei mehrmaliger Applikation - die Bildung Arzneimittel-abbauender Enzyme in der Leber induzieren (1). So führt beispielsweise Barbiturat-Vorbehandlung zu einer entsprechenden Induktion von Hydroxylasen und dadurch einerseits zu einem beschleunigten Abbau des Barbiturats selbst (2) und andererseits zu einer verstärkten Inaktivierung anderer Arzneimittel im Organismus (3). Diese Zusammenhänge sind fast ausschließlich durch Tierversuche, vorwiegend mit in-vitro Experimenten erschlossen worden. Einblicke in diese, für die Chemotherapie wichtigen Reaktionen in der Humanmedizin sind erschwert durch das Fehlen eines einfachen Verfahrens zum Nachweis der vermehrten Bildung Arzneimittel-abbauender Enzyme nach Arzneimittelbehandlung in-vivo.

In der vorliegenden Arbeit wird ein Analysen-Prinzip beschrieben, das den Nachweis von Hydroxylasen im lebenden Versuchstier und auch beim Menschen gestattet. Das Verfahren beruht. auf der Anwendung des von uns bereits früher aufgezeigten Prinzips der Stoffwechsel-labilen Tritium-Markierung $(5,6)$, hier auf Hydroxylierungsvorgänge bei Steroiden. Diese Steroidhydroxylierungen werden nämlich bevorzugt durch solche Enzyme hervorgerufen, die durch die Gabe von körperfremden Substanzen z. B. Barbituraten induziert werden (7).

\section{Methodik}

\section{Versuchstiere}

Es wurden männliche und weibliche Wistarratten eigener Zucht verwendet. Die Gewichte lagen zwischen 150-280 g. Die Tiere erhielten "Altromin" Standardfutter und Wasser ad libitum.

\section{Radioaktive Steroide}

Als Indikatorsubstanzen für die Anwesenheit Arzneimittelinduzierter Hydroxylasen wurden die radioaktiven Steroide: Ostradiol- $\left[6,7-{ }^{3} \mathrm{H}\right](415 \mathrm{mC} / \mathrm{mMol})$, Testosteron- $\left[7 \alpha-{ }^{3} \mathrm{H}\right](520 \mathrm{mC} /$ $\mathrm{mMol})$, Äthinylöstradiol-[6,7- $\left.{ }^{3} \mathrm{H}\right](500 \mathrm{mC} / \mathrm{mMol})$, und $\AA$ thinylnor-testosteronacetat-[7-3 $\left.{ }^{3} \mathrm{H}\right](780 \mathrm{mC} / \mathrm{mMol})$ verwendet. Alle radioaktiven Steroide stammen von der Firma Schering AG, Berlin. Sie wurden jeweils in $0,3 \mathrm{ml}$ Äthanol gelöst i. $\mathrm{m}$. injiziert. Jedes Tier erhielt etwa 5-15 $\mu \mathrm{g}$ mit $10-20 \mu \mathrm{C}$.

\section{Vorbebandlung}

Die mit Phenobarbital vorbehandelten Tiere erhielten zwei Tage lang zweimal täglich $40 \mathrm{mg} / \mathrm{kg}$ Phenobarbital. Das Phenobarbital wurde als freie Säure in äthanolischer Lösung i. p. injiziert. Die Kontrolltiere erhielten über den gleichen Zeitraum die entsprechenden Gaben des Lösungsmittels. Bei einer anderen Versuchsgruppe weiblicher Ratten (Stamm: Osborn-Mendel) wurde 5 Tage lang einmal täglich mit $5 \mathrm{mg} / \mathrm{kg}$ Phenobarbital-Na vorbehandelt.

\section{Messen der HTO-Bildung in Körperwasser}

Den Phenobarbital-vorbehandelten Tieren wurde meist am 5., z. T. am 6. Tag nach Versuchsbeginn, oder zum angegebenen

1) Diese Arbeit enthält teilweise Auszüge der unveröffentlichten Dissertationen von G. KöstrR und JuTrA HöNICKE. 
Zeitpunkt, das radioaktiv markierte Steroid injiziert. Anschließend wurden jewcils zwei Tiere in einen speziellen, luftdichten Käfig gesetzt, durch den getrocknete Luft geleitet wird. In einer Kühlfalle wurde dann das von den Tieren exspirierte Wasser kondensiert (5) und dessen spezifische Aktivität $(\mu \mathrm{C} / \mathrm{ml})$ im FlüssigSzintillationszähler gemessen. Durch Hochrechnung auf das gesamte Körperwasser wurde der HTO-Anteil und damit die Hydroxylierung im Gesamtorganismus in Prozent der injizierten Aktivität bestimmt. Für den Körperwasseranteil der Ratten wurden $68 \%$ des Körpergewichtes eingesetzt.

\section{Ergebnisse}

Injiziert man Ratten Östradiol-[6,7-3 $\mathrm{H}]$ oder Testosteron- $\left[7-{ }^{3} \mathrm{H}\right]$, so erscheint erwartungsgemä $\beta$ nur eine sehr geringe Tritium-Aktivität (etwa 1-3\%) im Körperwasser der Tiere $(5,8)$, da das Tritium bei beiden Hormonen stabil im Molekül gebunden ist (vgl. Kontrollen in Abb. 1 und 2) und die Steroide selbst nicht ins Atemwasser übertreten. Eine zweitätige Vorbehandlung mit Phenobarbital (zweimal täglich $40 \mathrm{mg}$ / $\mathrm{kg}$ ) fühtt $\mathrm{zu}$ keinem Anstieg des HTO-Spiegels im Körperwasser der Versuchstiere, wenn die Tritiummarkierten Steroide am 3. Versuchstag injiziert werden. Dagegen wurde nach einer zweitägigen Vorbehandlung der Tiere mit Phenobarbital (1. und 2. Tag des Versuchs) am 6. Tag nach Versuchsbeginn bei Injektion von Östradiol-[6,7-3 $\mathrm{H}]$ ein sehr starker Anstieg des HTO-Spiegels im Körperwasser der Versuchstiere gefunden. So erscheint bei männlichen Ratten 5 Stdn. nach Injektion des radioaktiven Östradiols etwa das 4 fache und bei weiblichen Ratten etwa das 5 fache des HTOGehaltes der Kontrolltiere im Atemwasser (Abb. 1). Den gleichen Effekt der Phenobarbital-Vorbehandlung erhält man mit Testosteron- $\left[7-{ }^{3} \mathrm{H}\right]$ als Indikatorsub-

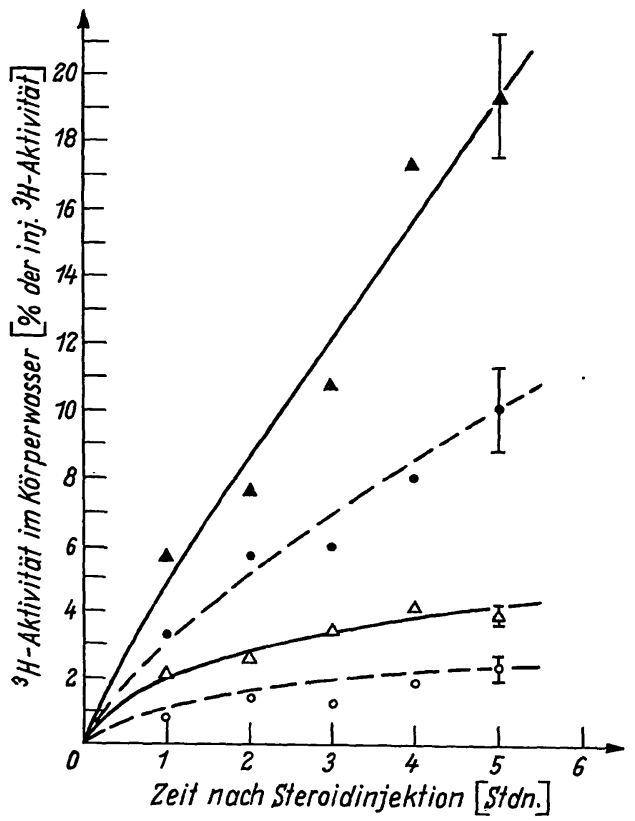

Abb. 1

HTO-Bildung in Ratten nach Injektion von Ostradiol- $\left[6,7-{ }^{-3} \mathrm{H}\right]$ ohne $\left(\Delta=\rho, \circ=\sigma^{\prime}\right)$ und nach $\left(\Delta=\%, \cdot=\sigma^{\prime}\right)$ Barbiturat-Vorbehandlung ( $\stackrel{2}{=} 3$ )

ng: 2 Tage zweimal tgl. $40 \mathrm{mg} / \mathrm{kg}$ (1. und

Barbiturat-Vorbehandlung: Tag des Versuchs)

Ostradiol-[6,7-3 H]-Inj. und Radiospirometrie: am 5. Tag des Versuchs Zum Zeitpunkt 5 Stdn. nach Injektion wurden Mittelwerte und Extremwerte aufgetragen

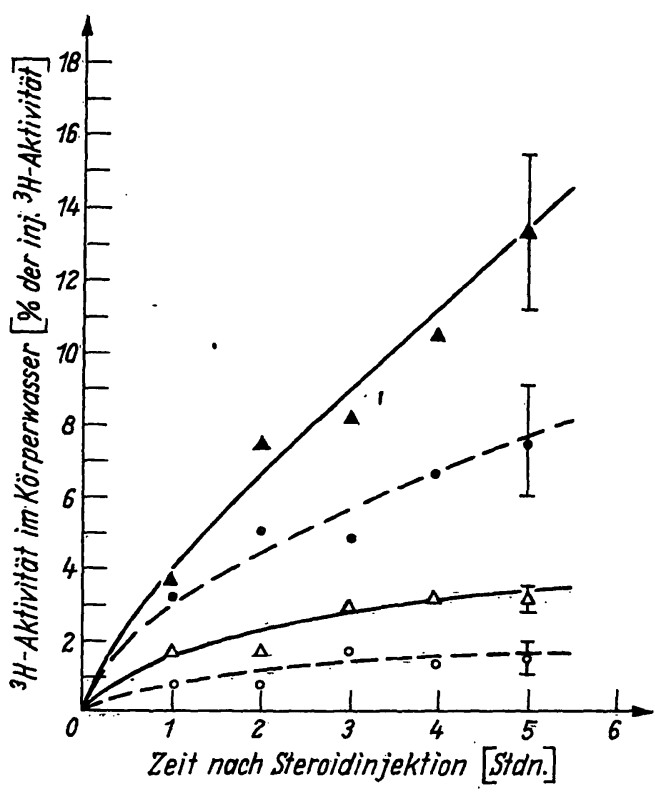

Abb. 2

HTO-Bildung in Ratten nach Injektion von Testosteron- $\left[7 \alpha^{-3} \mathrm{H}\right]$ ohne und nach Barbiturat-Vorbehandlung

Barbiturat-Vorbehandlung, Radiospirometrie und sonstige Bedingungen sowie Zeichenerklärung wie in Abb. 1

stanz. Auch hier wird - besonders bei weiblichen Tieren - das an sich im Testosteron stabil eingebaute Tritium in erheblichem Ausmaß labilisiert (Abb. 2).

Nach diesem positiven Ergebnis mit natürlichen Steroiden sollte untersucht werden, ob die induzierten $\mathrm{Hy}$ droxylasen auch auf in der Natur nicht vorkommende Steroide ansprechen.

Als Indikatorsteroid diente zunächst Äthinylöstrạdiol$\left[6,7-{ }^{3} \mathrm{H}\right]$, das viele Ovulationshemmer als östrogene Komponente enthalten. Der Versuch sollte außerdem klären, zu welchem Zeitpunkt nach Vorbehandlung das Steroid am stärksten hydroxyliert wird.

In 'Abbildung 3 ist die HTO-Bildung in Ratten nach Injektion von Äthinylöstradiol- $\left[6,7-{ }^{3} \mathrm{H}\right]$ ohne und nach Vorbehandlung mit Barbiturat aufgetragen. Während bei den: Kontrolltieren aus Äthinylöstradiol- $\left[6,7-{ }^{3} \mathrm{H}\right]$ $41 / 2$ Stdn. nach Injektion nur 0,8\% Tritium als HTO freigesetzt wird, steigt dieser Wert nach BarbituratVorbehandlung. Den höchsten Wert der HTO-Bildung mit 3\% erhält man 2 Tage nach Absetzen des Barbiturats (5. Versuchstag). Wird am 5. Versuchstag zusätzlich zum Indikatorsteroid nochmals Barbiturat $(1 \times 40$ $\mathrm{mg} / \mathrm{kg}$ ) injiziert, so fällt die HTO-Bildung auf $1,2 \%$ (Abb. 3).

Auch bei Äthinyl-nor-testosteron-acetat- $\left[7-{ }^{3} \mathrm{H}\right]$, der gebräuchlichsten gestagenen Komponente von Ovulationshemmern, konnte nach Barbiturat-Behandlung eine erhöhte HTO-Bildung und damit Hydroxylierung festgestellt werden. Während bei männlichen Ratten als Kontrolltiere nur 0,8\% HTO 5 Stdn. nach Steroidinjektion gefunden werden, erhält man nach BarbituratBehandlung $2 \%$ HTO. Interessanterweise ist dagegen bei Rattenweibchen nach Barbituratgabe keine vermehrte H'TO-Bildung mit Äthinyl-nor-testosteron-acetat- $\left[7-{ }^{3} \mathrm{H}\right]$ als Indikatorsteroid nachweisbar. 


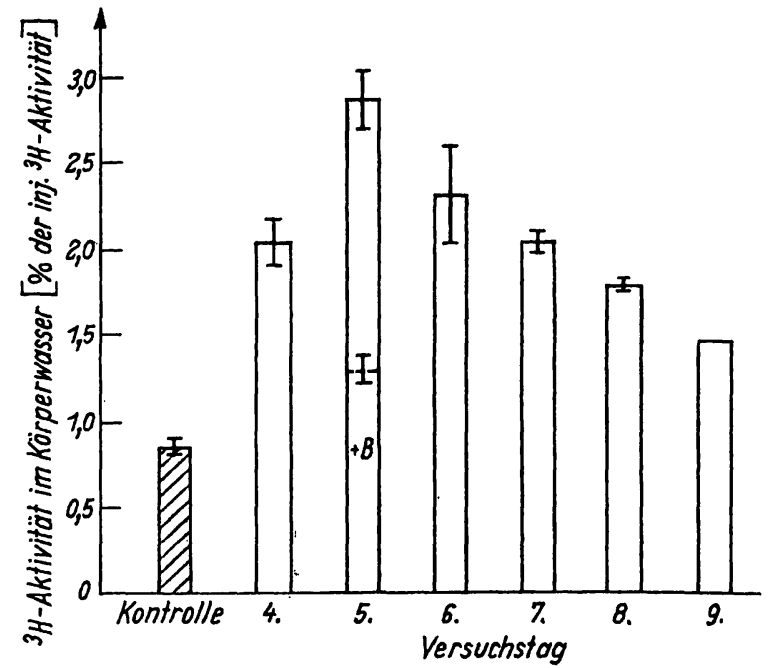

Abb. 3

HTO-Bildung in Rattenweibchen nach Injektion von Äthinylöstradiol$[6,7-\mathrm{s} H]$ in Abhängigkeit vom zeitlichen Abstand zur BarbituratVorbehandlung

Kontrollen: Schraffierte Säule (Mittelwert aus 3 Messungen mit Barbiturat-

Vorbehandlung: 2 Tage zweimal tgl. $40 \mathrm{mg} / \mathrm{kg}$ (1. und 2. Tag des Athinylöstradiol-

$\left[6,7-{ }^{3} \mathrm{H}\right]-$ Inj. und

Radiospirometrie: am 4.-9. Tag des Versuchs. HTO-Werte 4,5 Stdn. nach Athinylöstradiolinjektion.

Mittelwerte aus Doppelbestimmungen mit Extremwerten.

Gestrichelte Linie am 5. Tag zusätzliche Barbituratgabe (40 mg/kg)

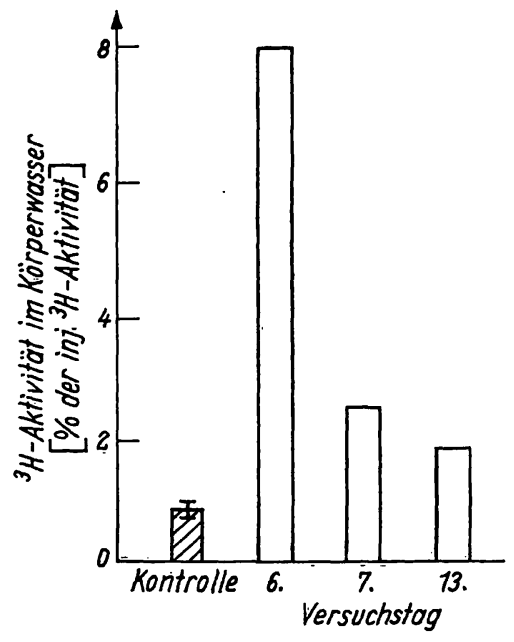

Abb. 4

HTO-Bildung in Ratten nach Ostradiol-[6,7-8 H]-Injektion in Abhängigkeit vom zeitlichen Abstand zur Barbiturat-Vorbehandlung Kontrollen: Schraffierte Säule (Mittelwerte aus 5 Messungen mit Barbituratmittlerem Fehler des Einzelwertes).

Vorbehandlung: 5 Tage tgl. mit $5 \mathrm{mg} / \mathrm{kg}(1 .-5$. Tag des Versuchs) Radiospirometrie: Am 6., 7. und 13. Tag des Versuchs. HTO-Werte 2,5 Stdn. nach Ostradiól-Injektion.

$\mathrm{Zu}$ diesem Versuch wurden Osborn-Mendel Rattenweibchen mit einem Tiergewicht von $140-170 \mathrm{~g}$ verwendet

Auch bei einer Vorbehandlung der Versuchstiere mit wesentlich geringeren Dosen von Phenobarbital findet man einen erhöhten HTO-Spiegel im Körperwasser nach Injektion von Östradiol-[6,7-3 $\mathrm{H}]$. Entsprechend den in Abbildung 3 niedergelegten Befunden mit Äthinylöstradiol- $\left[6,7-{ }^{3} \mathrm{H}\right]$ und den Ergebnissen von in-vitro Untersuchungen anderer Autoren (1), nimmt auch hier die HTO-Bildung mit zunehmender Zeitdifferenz zwischen Ende der Barbiturat-Vorbehandlung und Injektion der Indikatorsteroide ab (Abb. 4).

\section{Diskussion}

Unsere Kontrollversuche zeigen nur eine sehr geringfügige HTO-Bildung nach Injektion von Testosteron$\left[7 \alpha-{ }^{3} \mathrm{H}\right]$, Östradiol- $\left[6,7-{ }^{3} \mathrm{H}\right]$, Äthinylöstradiol- $\left[6,7-{ }^{3} \mathrm{H}\right]$ und Äthinyl-nor-testosteron-acetat-[7-3 $\mathrm{H}]$. Die Hydroxylierung an $\mathrm{C}_{7}$ und/oder $\mathrm{C}_{6}$ der injizierten Tritiummarkierten Steroide ist also sehr gering.

Dagegen findet man nach Vorbehandlung mit Barbiturat einen exheblichen Anstieg der HTO-Bildung, den wir auf die bekannte Induktion von Steroidhydroxylasen in der Leber der Versuchstiere nach Barbituratgabe zurückführen. So werden nach Barbituratgabe Östradiol und Testosteron innerhalb von $5 \mathrm{Stdn}$. bei Weibchen zu 15-20\% und bei Männchen etwa zu 10\% hydroxyliert. Bei den zwei in Ovulationshemmern gebräuchlichen Steroiden tritt unter den gleichen Bedingungen nur eine Hydroxylierung von $2-3 \%$ auf. $\mathrm{Da}$ die hydroxylierten Hormone fast keine biologische Aktivität mehr besitzen, bewirkt also die Induktion von Hydroxylasen teilweise eine erhebliche, irreversible Inaktivierung dieser Steroide.

Wie bei den von anderer Seite ausgeführten in-vitro Untersuchungen (1) zeigt auch unsere Methode in-vivo, $\mathrm{da} B$ die induzierten Hydroxylasen erst nach einer gewissen Inkubationszeit nach Barbituratgabe nachweisbar sind.

Ebenfalls wie bei den in-vitro Untersuchungen (1), werden einmal durch Barbiturat induzierte Hydroxylasen durch nochmalige Barbituratgabe gehemmt (vgl. in Abb. 3 die Werte am 5. Versuchstag). Steroid und Barbiturat konkurrieren also um die induzierten $\mathrm{Hy}-$ droxylasen. So läßt sich zwanglos die verminderte HTO-Ausbeute bei Barbiturat-vorbehandelten Ratten nach Östradiol-[6,7-3H] Injektion bei gleichzeitiger, nochmaliger Barbituratgabe erklären.

Das Auftreten von Tritium im Atemwasser nach Injektion der Tritium-markierten Steroide z. B. von Testosteron- $\left[7-{ }^{3} \mathrm{H}\right]$ erklären wir uns entsprechend dem folgenden Schema:

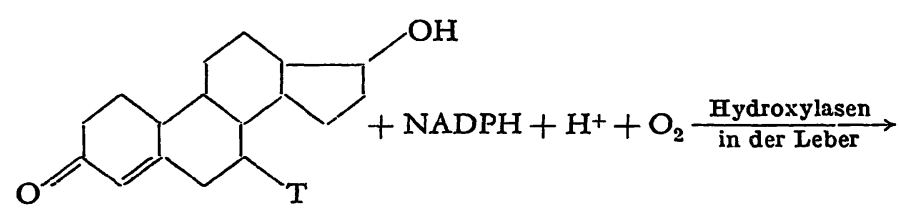

Testosteron $\left[7 \alpha^{-3} \mathrm{H}\right]$

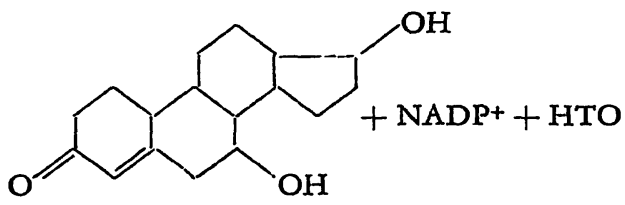

$7 \alpha$-Hydroxytestosteron

HTO in der Leber $\stackrel{\text { Kreislauf }}{\longrightarrow}$ HTO homogen im Körperwasser verteilt 
Da man aus dem Körpergewicht auf die Menge an Körperwasser schließen kann, ist es mit dieser Methode möglich, das Ausmaß der Hydroxylierung im Gesamtorganismus zu bestimmen. Man ist nicht auf den Anteil in den Körperausscheidungen beschränkt, wie es der Fall wäre, wenn man die Hydroxylierung durch die mühsame und stets unvollständige Isolierung der einzelnen hydroxylierten Steroide aus Harn und Kot bestimmen wollte.

Bei Betrachtung der Absolutumsätze muß natürlich der Isotopieeffekt berücksichtigt werden, der zu einem verlangsamten Umsatz des Tritium-markierten Steroids gegenüber dem nicht markierten Steroid führen dürfte. Für die hier gemessenen Effekte der Barbiturat-Vorbehandlung kann allerdings dieser Gesichtspunkt außer acht gelassen werden, da wir stets den Umsatz von Tritium-markierten Steroiden untereinander vergleichen (z. B. Hydroxylierung von Testosteron- $\left[7 \alpha-{ }^{3} \mathrm{H}[\right.$ vor und nach Barbiturat-Behandlung).

Das Ausmaß der Hydroxylierung errechnet sich aus folgender Formel:

$\mu \mathrm{C}$ pro $\mathrm{ml}$ Atemwasser $\cdot 100$

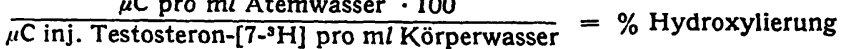

Dabei wird vorausgesetzt, daß bei der Einführung der Hydroxylgruppe in Position 7 von den beiden an $\mathrm{C}_{7}$ gebundenen $\mathrm{H}$-Atomen stets das in $\alpha$-Stellung stehende radioaktive Wasserstoffatom substituiert wird. Das ist nach den Untersuchungen von CONNEY und KцUтсн (7), seht wahrscheinlich: Mit Lebermikrosomen von Barbiturat-behandelten Rattenmännchen als Enzymquelle entsteht bei der Hydroxylierung von Testosteron hauptsächlich $7 \propto$ Hydroxytestosteron.

Wenn wir annehmen, daß bei der Herstellung des Östradiol- $\left[6,7-{ }^{3} \mathrm{H}\right]$ bzw. des tritierten Äthinylöstradiols keine der vier möglichen stereoisomeren Formen bevorzugt wird, sondern daß diese mit statistischer Wahrscheinlichkeit auftreten, so müssen wir bei der Applikation von Östradiol- $\left[6,7-{ }^{3} \mathrm{H}\right]$ damit rechnen, daß bei der Hydroxylierung nicht jeden Steroidmoleküls ein Tritium-Atom im Wasser erscheint. Das bedeutet: Das Ausmaß der Hydroxylierung an $C_{6}$ oder $C_{7}$ kann beim Östradiol- $\left[6,7-{ }^{3} \mathrm{H}\right]$ größer sein, als wir es mit unserer Methode nachweisen können. Wir erfassen also nur Mindestwerte.

In-vitro Versuche von Breuer und Mitarbeitern (9) mit Rattenleber haben gezeigt, daß beim Ostradiol sowohl eine Hydroxylierung in $6 \alpha$-als auch in $6 \beta$-Position möglich ist.
Auch ist es beim Östradiol-[6,7-3H] nicht möglich zu entscheiden, ob dieses Steroid Tritium an Position 6 und 7 abgibt, falls ein Dihydroxysteroid entsteht.

Nach den Ergebnissen unserer Versuche halten wir das nicht für unwahrscheinlich, weil die Ausbeute an HTO bei Gabe von Östradiol-[6,7-3 H] deutlich größer ist als bei Gabe von Testosteron-[7-3 $\mathrm{H}]$.

Abschließend seien einige allgemeinere Gesichtspunkte diskutiert: Das hier aufgezeigte Nachweisverfahren für Hydroxylierungen innerhalb dẹs intakten Organismus ist eine Variante der Stoffwechsel-labilen TritiumMarkierung (5) insofern, als das stabil in das Kohlenstoffgerüst des Steroids gebundene Tritium durch eine Stoffwechselleistung aus dem Molekül entfernt wird. $\mathrm{Da}$ aber diese Reaktion im Organismus unserer Versuchstiere (aber auch beim Menschen) normalerweise kaum, sondern nur nach Arzneimittel-Behandlung abläuft, können wir hier nur von einer „,bedingt Stoffwechsel-labilen" Tritium-Markierung beim Testosteron[7-3 $\mathrm{H}]$ oder beim Östradiol- $\left[6,7-{ }^{3} \mathrm{H}\right]$ sprechen.

Die Methode ist prinzipiell zum in-vivo Nachweis einer Vielzahl von Hydroxylierungsvorgängen geeignet, falls die entsprechenden Tritium oder Deuterium ${ }^{2}$ ) markierten Substrate zur Verfüguing stehen.

Man beachte aber, daß die HTO-Bildung nur Hydroxylierungen an dem C-Atom des betreffenden Moleküls anzeigt, welches das Tritium-Atom trägt. Hydroxylierungen an nicht Tritium-markierten Stellen des Moleküls sind dieser Spezifität wegen durch HTO-Bestimmung nicht nachweisbar.

Wie wir in früheren Untersuchungen gezeigt haben, ist die Analyse von Körperwasserproben beim Menschen durch Destillation von Harn- oder Blutproben ohne Schwierigkeiten möglich (6). Daher ist das hier aufgezeigte Verfahren auch in der Humanmedizin anwendbar, wo seine Vorteile besonders deutlich und Untersuchungen über die gegenseitige Beeinflussung von Arzneimitteln besonders wichtig sind. Uber derartige Versuche wird an anderer Stelle berichtet.

Für materielle Unterstützung danken wir der Firma Schering A.G., Berlin.

2) Bei Deuterium-markierten Verbindungen muß im Körperwasser der HDO-Gehalt bestimmt werden.

\section{Literatur}

1. Conney, A., Pharmacol. Rev., Baltimore 19, 317 (1967). 2. ReMmer, H., Arch. internat. pharmacodyn. thérap. 152, 346 (1964). - 3. Remmer, H. und M. Siegert, Arch. exp. Pathol. Pharmakol. 247, 522 (1964). - 4. KuntzMan, R., M. JaCOBson, K. Schnerdman und A. Conney, J. Pharmacol. Exper. Therap., Baltimote 146, 280 (1964). - 5. WenzeL, M., H. KLEUCKER und
P. E. Schulze, Z. Naturforsch. $21 b, 1178$ (1966). - 6. WENZEL, M. und K. U. BLuM, diese Z. 5, 202 (1967). - 7. ConNEx, A. und A. KuUTCH, J. biol. Chemistry 238, 1611 (1963). - 8. WENZEL, M., L. Pitzel und P. E. Schulze, Hoppe-Seyler's Zschr. physiol. Chem. 350, 341 (1969). - 9. BReuER, H., R. KNUPPEN und G. Pangels, Biochim. biophysica Acta (Amsterdam) 65, 1 (1962).

Prof. Dr. M. Wenzel 1000 Berlin 33

Königin-Luise-Str. $2-4$ 